\title{
DILUTE GAS-LIQUID FLOWS WITH LIQUID FILMS ON WALLS
}

\author{
John MORUD ${ }^{1}$ \\ ${ }^{1}$ SINTEF Materials and Chemistry, 7465 Trondheim, NORWAY
}

\begin{abstract}
A model for dilute gas-liquid flows with films at the walls has been implemented in the CFD code FLUENT. The model consists of transport equations for droplets in the gas and for liquid transport in the film. Exchange of liquid between the two occurs by atomization of the film and by droplet deposition. Predictions are in reasonable agreement with known cases, such as horizontal and vertical pipe flows. However, independent validation of the code is yet to be performed.
\end{abstract}

\section{NOMENCLATURE}

$\begin{array}{ll}\mathbf{a} & \text { Fluid acceleration } \\ C & \text { Droplet concentration } \\ C_{f, p i p e} & \text { Friction factor for pipe } \\ d_{d r o p} & \text { Droplet diameter } \\ d_{*} & \text { Dimensionless droplet diameter } \\ D & \text { Droplet diffusion coefficient } \\ \mathbf{g} & \text { Gravity } \\ h & \text { Film thickness } \\ k & \text { Turbulent kinetic energy } \\ k_{A}^{\prime} & \text { Constant in atomization model } \\ R_{A} & \text { Atomization rate } \\ R_{D} & \text { Deposition rate } \\ S c_{t} & \text { Turbulent Schmidt number } \\ \mathbf{u} & \text { Velocity } \\ U_{g a s} & \text { Gas velocity in pipe } \\ u^{*} & \text { Shear velocity } \\ u_{*} & \text { Dimensionless settling velocity } \\ V_{d r o p} & \text { Droplet impaction velocity } \\ V_{w} & \text { Deposition velocity }\end{array}$

Greek:

$\gamma \quad$ Parameter in Csanady model

$\Gamma \quad$ Film mass flux

$\Gamma_{\text {crit }} \quad$ Critical film mass flux

$\mu \quad$ Viscosity or statistical mean

$\mu_{\mathrm{t}} \quad$ Turbulent viscosity

$\rho \quad$ Density

$\begin{array}{ll}\sigma & \text { Surface tension or standard deviation } \\ \tau_{\text {wall }} & \text { Wall shear stress } \\ \tau+ & \text { Dimensionless relaxation time }\end{array}$

Subscripts:

2D Two-dimensional operator

drop Droplet phase

gas Gas phase

liq Liquid phase

$t \quad$ tangential or terminal

wall At the wall

\section{INTRODUCTION}

In many two-phase gas/liquid flows, liquid films at walls are important. Examples are condensation in heat exchangers; annular flow in pipes; and inlet manifolds for chemical reactors with two-phase feed. However, the leading CFD codes are not presently able to calculate liquid films. The objective of the present work was to develop and implement a model for liquid films at walls; a model for droplet transport in gas flows; together with relations for the interaction between the two. The interactions are the deposition of droplets at walls and atomization/reentrainment of liquid from the liquid film into the gas due to Kelvin-Helmholtz instabilities.

There has been a significant amount of papers on wall films, most of which are on annular flows in pipes. This paper is heavily influenced by the work of Pan and Hanratty (2002), who studied entrainment in horizontal annular pipe flow. For droplet diffusion, the papers by Loth (2001), Mols and Oliemans (1998) and by Mols et. al. (2000) have been particularly useful. For droplet deposition at walls, the results of McCoy and Hanratty (1997) has been used.

A brief outline of the paper: First, the transport models for liquid films and droplet transport are described, together with models for droplet deposition and film atomization. Then, an example calculation for air/water flow in a horizontal pipe segment is given. Finally, possible extensions are outlined. 


\section{MODEL DESCRIPTION}

\section{Model overview}

The present model consists of the following elements:

- A two-dimensional transport equation for films at walls. The film is assumed thin compared to the dimensions of the flow geometry.

- A transport equation for droplets in a bulk gas phase.

- A relation for the deposition and entrainment of liquid between the film and the bulk phase.

In the following, these elements are described in detail. Note that the sub models for the film and the droplet phase are independent in the sense that the film model could be used together with other models for the droplet phase.

\section{Film mode}

The following two-dimensional transport equation is used for the film on a surface ${ }^{1}$ :

$$
\frac{\partial \rho_{l i q} h}{\partial t}+\nabla_{2 D} \cdot\left(\rho_{\text {liq }} h \mathbf{u}_{\text {film }}\right)=R_{D}-R_{A}
$$

Here, $\rho_{\text {liq }}\left[\mathrm{kg} / \mathrm{m}^{3}\right]$ is the liquid density, $h[\mathrm{~m}]$ is the film thickness, $R_{D}\left[\mathrm{~kg} / \mathrm{m}^{2} \mathrm{~s}\right]$ is the rate of droplet deposition, and $R_{A}\left[\mathrm{~kg} / \mathrm{m}^{2} \mathrm{~s}\right]$ is the rate of atomization of the film. The film velocity, $\mathbf{u}_{\text {film }}[\mathrm{m} / \mathrm{s}]$ is calculated from:

$\mathbf{u}_{\text {film }}=\frac{|h| \boldsymbol{\tau}_{\text {wall }}}{2 \mu_{\text {liq }}}+\frac{h^{2} \mathbf{g}_{\mathrm{t}}}{3 \mu_{\text {liq }}}$

Here, $\tau_{\text {wall }}\left[\mathrm{N} / \mathrm{m}^{2}\right]$ is the wall shear stress taken as a vector and $\mathbf{g}_{\mathbf{t}}\left[\mathrm{m} / \mathrm{s}^{2}\right]$ is the component of gravity tangential to the surface.

\section{Droplet transport}

In the present FLUENT implementation, a simple driftflux type of equation for the droplet phase is implemented

$$
\frac{\partial C}{\partial t}+\nabla \cdot\left(C \mathbf{u}_{d r o p}-D \nabla C\right)=0
$$

where $C\left[\mathrm{~kg} / \mathrm{m}^{3}\right]$ is the droplet concentration in the gas, $\mathbf{u}_{\text {drop }}$ is the droplet velocity and $D\left[\mathrm{~m}^{2} / \mathrm{s}\right]$ is the droplet diffusion coefficient described below. This model is intended as a stepping stone for later models for droplet size distributions. Presently, there is only one droplet size.

\section{Droplet velocity}

The droplet velocity, $\mathbf{u}_{\mathrm{drop}}$, is calculated as follows. It is assumed that the droplet relaxation time is much lower than a characteristic (lagrangean) time scale of the mean flow. The calculations are based on a correlation for settling velocity by Turton and Clark (1987). The correlation is explicit, and gives the terminal velocity of

\footnotetext{
${ }^{1}$ To emphasize the two-dimensional nature of the equation, a "2D" subscript has been added to the divergence operator. The wall is thus considered to be a two-dimensional manifold.
}

falling spheres to within $\sim 8 \%$ of the one calculated from the standard drag curve.

A dimensionless particle diameter is given by

$$
d_{*}=d_{\text {drop }} \frac{\left[\|\mathbf{g}-\mathbf{a}\| \rho_{\text {gas }}\left(\rho_{\text {liq }}-\rho_{\text {gas }}\right)\right]^{1 / 3}}{\mu_{\text {gas }}^{2}}
$$

where $\mathbf{g}\left[\mathrm{m} / \mathrm{s}^{2}\right]$ is the gravity and $\mathbf{a}=\mathbf{D u} / \mathbf{D t}$ is the acceleration of the gas phase.

The Turton-Clark correlation gives a settling velocity on dimensionless form:

$u_{*}=\left[\left(\frac{18}{d_{*}^{2}}\right)^{0.824}+\left(\frac{0.321}{d_{*}}\right)^{0.412}\right]^{-1.214}$

(5)

Finally, the settling velocity, $u_{t}$, is calculated from

$$
u_{*}=u_{t}\left[\frac{\rho_{\text {gas }}^{2}}{\|\mathbf{g}-\mathbf{a}\| \mu_{\text {gas }}\left(\rho_{\text {liq }}-\rho_{\text {gas }}\right)}\right]^{1 / 3}
$$

The drift between the droplets and the gas is then calculated from

$$
\mathbf{u}_{d r o p}-\mathbf{u}_{g a s}=u_{t} \frac{(\mathbf{g}-\mathbf{a})}{\|\mathbf{g}-\mathbf{a}\|}
$$

\section{Droplet diffusion}

Rigorous models for droplet diffusion should take into account crossing trajectory, inertial-limit and continuity effects. In the present model, we use the transverse diffusion coeffisient of the Csanady (1963) model. With "standard" parameters, this equates to :

$D=\frac{\mu_{t} / S c_{t}}{\sqrt{1+0.7 \gamma^{2}}}$
$\gamma=\frac{\left\|\mathbf{u}_{\text {drop }}-\mathbf{u}_{\text {gas }}\right\|}{\sqrt{2 k / 3}}$

\section{Atomization of the film}

The model for atomization of the liquid film follows the work by Pan and Hanratty (2002). The situation is depicted in Figure 1. 


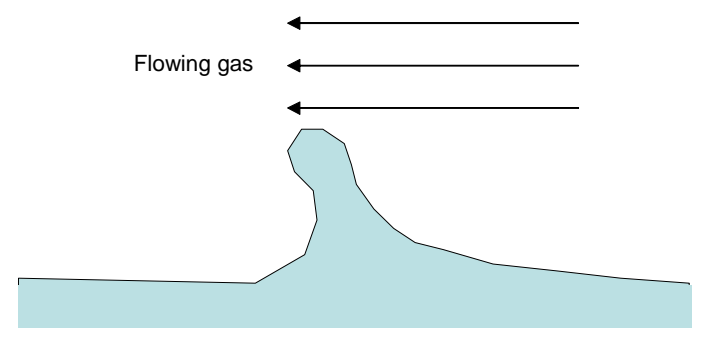

Figure 1: Atomization of liquid film.

Due to the passing gas flow, the liquid film is unstable (Kelvin-Helmholz mechanism), and droplets are torn off the forming waves. For pipes, a correlation for the total rate of liquid entrainment, $R_{A}\left[\mathrm{~kg} / \mathrm{m}^{2} \mathrm{~s}\right]$, into the gas is given by:

$R_{A}=\frac{k_{A}^{\prime} U_{\text {gas }}^{2} \sqrt{\rho_{\text {gas }} \rho_{\text {liq }}}}{\sigma}\left(\Gamma-\Gamma_{\text {crit }}\right)$

where $\Gamma[\mathrm{kg} / \mathrm{m} / \mathrm{s}]$ is the liquid film mass flow rate per meter circumference and $\Gamma_{\text {crit }}$ is a critical flow rate. Below $\Gamma_{\text {crit }}$ there is no atomization. The constant $k_{A}^{\prime}$ is approximately $3 \cdot 10^{-6}$ (Pan/Hanratty 2002).

For CFD calculations in general geometries, the form of the Pan/Hanratty (2002) correlation needs some modification. If we define at friction coefficient for the pipe as

$C_{f, p i p e}=\frac{\tau_{\text {wall }}}{1 / 2 \rho_{\text {gas }} U_{\text {gas }}^{2}}$

we can eliminate the presence of the gas velocity, and obtain:

$R_{A}=\frac{k_{A}^{\prime} \tau_{\text {wall }} \sqrt{\rho_{\text {gas }} \rho_{\text {liq }}}}{1 / 2 \rho_{\text {gas }} C_{f, \text { pipe }} \sigma}\left(\Gamma-\Gamma_{\text {crit }}\right)$

which is the one implemented in FLUENT. For pipe flows, $\mathrm{C}_{\mathrm{f} \text {,pipe }}$ may be looked up in a standard Moody chart.

The critical film flow, $\Gamma_{\text {crit }}$, is given $b^{2}$ :

$\Gamma_{\text {crit }}=\frac{1}{4} \mu_{\text {liq }} \cdot \operatorname{Re}_{L F C}$, where:

$\operatorname{Re}_{L F C}=7.3 \log (\omega)^{3}+44.2 \log (\omega)^{2}-263 \log (\omega)+439$,

$\omega \equiv \frac{\mu_{\text {liq }}}{\mu_{\text {gas }}} \sqrt{\frac{\rho_{\text {gas }}}{\rho_{\text {liq }}}}, \quad$ validity $(1.8<\omega<28)$

\section{Droplet deposition onto the film}

The deposition rate of droplets, $R_{D}\left[\mathrm{~kg} / \mathrm{m}^{2} \mathrm{~s}\right]$, onto the film is taken as

$\mathrm{R}_{\mathrm{D}}=\mathrm{V}_{\mathrm{w}} \mathrm{C}$

where $C$ is the droplet concentration at the wall and $V_{w}$ is the mean impaction velocity of droplets hitting the wall.

To model $V_{w}$, we do the following reasoning, similar to Pan/Hanratty (2002):

\footnotetext{
${ }^{2}$ Note that the entrainment rate is zero if $\Gamma<\Gamma$ crit. In that case, the film is stable.
}

The velocity, $V_{d r o p}$, of individual droplets in the direction normal to the wall is assumed to be normally distributed with mean $\mu$, which is taken as the settling velocity in the direction normal to the wall, and a standard deviation $\sigma$ which is yet to be determined. $V_{w}$ is then the expected velocity towards the wall:

$V_{w}=\int_{0}^{\infty} V_{d r o p} \cdot f\left(V_{d r o p}\right) \cdot d V_{d r o p}$

(14)

Here $f($.$) is the Gaussian velocity distribution. This$ integral has an analytic solution:

$\mathrm{V}_{\mathrm{w}}=\frac{1+\operatorname{erf}(\mu / \sqrt{2} \sigma)}{2} \cdot \mu+\frac{e^{-\frac{\mu^{2}}{2 \sigma^{2}}}}{\sqrt{2 \pi}} \cdot \sigma$

which is the form implemented. Note that equation (15) is a general solution for a Gaussian distribution with mean velocity $\mu$ and standard deviation $\sigma$, where in the present case $\mu=0$.

It remains to find the standard deviation, $\sigma$. For vertical pipes, we have the following correlation (McCoy and Hanratty 1997)

$V_{w} / u^{*}=1.3 \cdot 10^{-5} \quad$ for $\tau^{+}<0.2$

$V_{w} / u^{*}=3.25 \cdot 10^{-4}\left(\tau^{+}\right)^{2}$ for $0.2<\tau^{+}<22.9$

$V_{w} / u^{*}=0.17 \quad$ for $22.9<\tau^{+}<14751$

$V_{w} / u^{*}=20.7 / \sqrt{\tau^{+}} \quad$ for $\tau^{+}>14751$

where

$\tau^{+}=\frac{1}{18}\left(\frac{d_{d r o p} \rho_{\text {gas }} u^{*}}{\mu_{\text {gas }}}\right)^{2} \frac{\rho_{\text {drop }}}{\rho_{\text {gas }}}$

is a dimensionless droplet relaxation time and $u^{*}[\mathrm{~m} / \mathrm{s}]$ is the shear velocity.

For this particular case, we have $\mathrm{V}_{\mathrm{w}}=\sigma / \sqrt{2 \pi}$. Assuming that the standard deviation, $\sigma$, is the same for other wall inclinations, we get the following correlation for the general case:

$$
\begin{array}{ll}
\sigma / u^{*}=\sqrt{2 \pi} \cdot 1.3 \cdot 10^{-5} & \text { for } \tau^{+}<0.2 \\
\sigma / u^{*}=\sqrt{2 \pi} \cdot 3.25 \cdot 10^{-4}\left(\tau^{+}\right)^{2} & \text { for } 0.2<\tau^{+}<22.9 \\
\sigma / u^{*}=\sqrt{2 \pi} \cdot 0.17 & \text { for } 22.9<\tau^{+}<14751 \\
\sigma / u^{*}=\sqrt{2 \pi} \cdot 20.7 / \sqrt{\tau^{+}} & \text {for } \tau^{+}>14751
\end{array}
$$

which is the one implemented.

\section{EXAMPLE CALCULATION}

The model has been implemented in FLUENT using UserDefined-Functions (UDF).

As an example, flow in a horizontal pipe segment is calculated. The input data is for small water droplets in air, as given in Table 1 . 


\begin{tabular}{|lll|}
\hline Description & Value & \\
\hline Pipe diameter $(2 \mathrm{ft})$ & 0.67 & $\mathrm{~m}$ \\
Pipe length & 10.0 & $\mathrm{~m}$ \\
Roughness height & $6.7 \cdot 10^{-4}$ & $\mathrm{~m}$ \\
& & \\
Inlet velocity & 10.0 & $\mathrm{~m} / \mathrm{s}$ \\
Turbulent intensity & 10.0 & $\%$ \\
Inlet droplet concentration & 0.01 & $\mathrm{~kg} / \mathrm{m}^{3}$ \\
& & \\
Gas density & 1.2 & $\mathrm{~kg} / \mathrm{m}^{3}$ \\
Gas viscosity & $1.15 \cdot 10^{-5}$ & $\mathrm{~Pa} \cdot \mathrm{s}$ \\
Liquid density & 1000 & $\mathrm{~kg} / \mathrm{m}^{3}$ \\
Liquid viscosity & 0.001 & $\mathrm{~Pa} \cdot \mathrm{s}$ \\
Surface tension & 0.075 & $\mathrm{~N} / \mathrm{m}$ \\
Droplet diameter & 50 & $\mu \mathrm{m}$ \\
\hline
\end{tabular}

Table 1: Simulated conditions.

\section{Calculated results}

In the following, results are presented for:

- Droplet concentration field

- Film thickness at walls

- Film velocity near the inlet

- Film mass flux

\section{Droplet concentration field}

Figure 2 shows contours of droplet concentration at pipe cross sections. The inlet is to the left.

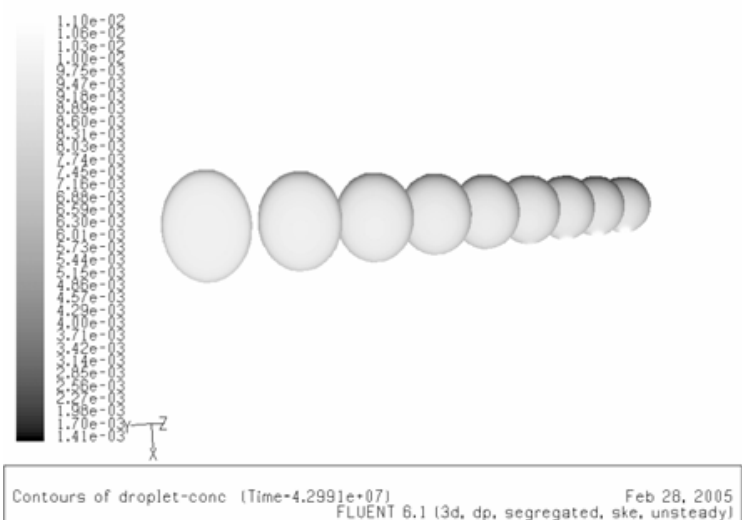

Figure 2: Droplet concentration, $\left[\mathrm{kg} / \mathrm{m}^{3}\right]$, in pipe cross sections

The effect of the gravity is apparent; the droplets are drawn downwards, leading to an accumulation of droplets at the lower part of the pipe.

In order to see this better, the concentration field is drawn in Figure 3 in a vertical slice through the pipe axis:

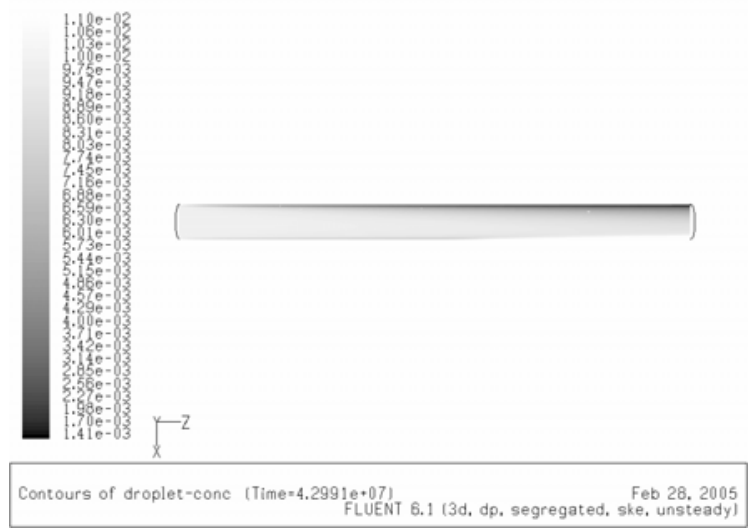

Figure 3: Droplet concentration, $\left[\mathrm{kg} / \mathrm{m}^{3}\right]$, in vertical slice through the pipe axis. Pipe inlet to the left.

\section{Film thickness}

Figure 4 shows contours of film thickness at the pipe wall as seen from the pipe inlet into the pipe. As can be seen, liquid tends to accumulate at the bottom of the pipe. Also, minor ringing artifacts may be seen due to the nondissipative nature of the model, but the ringing is tolerable.

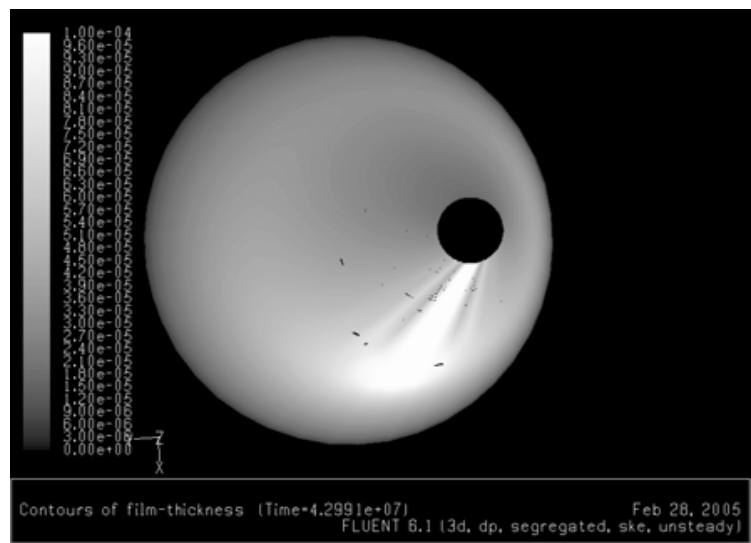

Figure 4: Film thickness, [m], at wall

In order to see the film thickness better, xy-plots are shown in Figure 5. The curves apply to:

- The top of the pipe, i.e. the line parallel to the axis, but one pipe radius above it.

- The bottom of the pipe

- The sides of the pipe, i.e. at lines parallel to the axis, but shifted $+/$ - one radius sideways. 


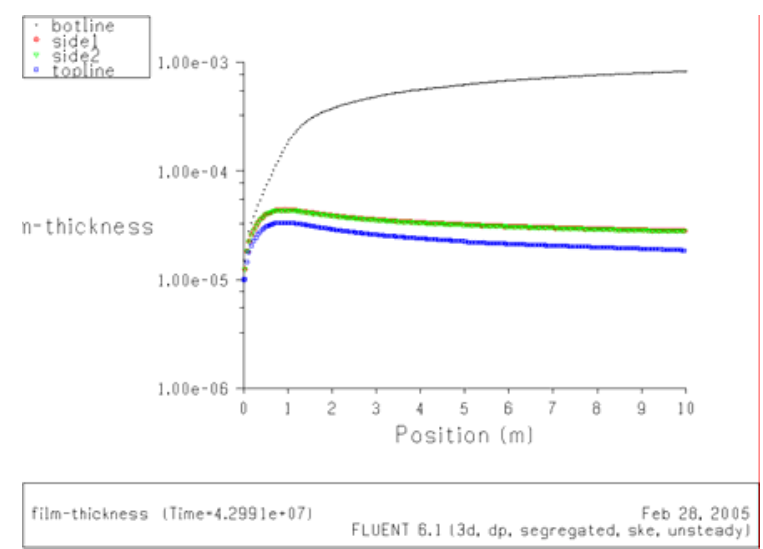

Figure 5: Film thickness, $[\mathrm{m}]$, as function of axial position. The curves apply to i) the bottom of the pipe, ii) the sides of the pipe and iii) the top of the pipe.

\section{Film velocity}

Figure 6 shows the film velocity near the pipe inlet, which is of the order of $1 \mathrm{~cm} / \mathrm{s}$. The effects of gravity on the film is apparent.

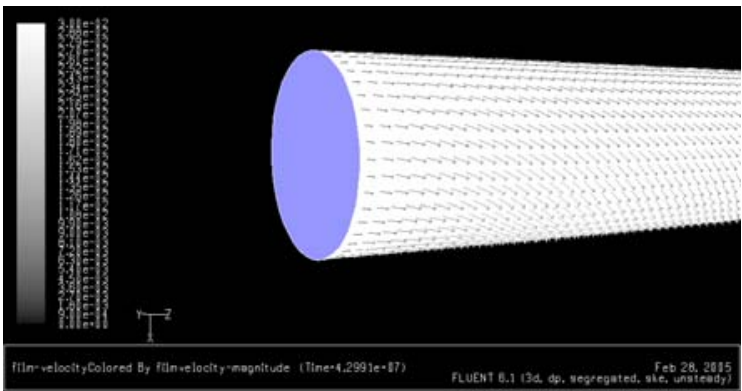

Figure 6: Film velocity, $[\mathrm{m} / \mathrm{s}]$ near the pipe inlet

\section{Film mass flux}

Figure 7 shows the xy-plots of the calculated film mass flux at the top/side/bottom of the pipe.

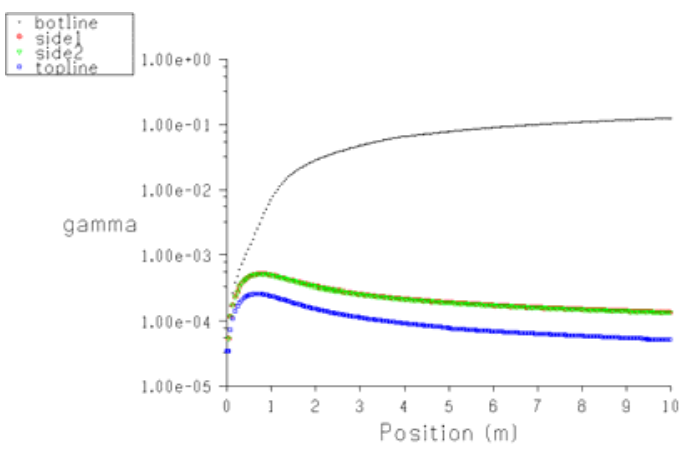

gamma (Time-4.2991 e*07)

FLUENT 6.1 (30, dp, segregated, ske, 28,2005 unsteady)

Figure 7: Film mass flux, $[\mathrm{kg} / \mathrm{m} . \mathrm{s}]$, as function of axial position.

Again, the liquid accumulation at the bottom is apparent.

\section{DISCUSSION}

\section{Experimental validation}

Presently, the model has only been benchmarked against known results and existing models for special cases, such as annular flows in horizontal and vertical pipes. Thus, the model needs independent experimental validation before one may be certain about the accuracy. However, the submodels for entrainment and deposition are validated in the literature (e.g. Pan/Hanratty 2002).

\section{Choice of droplet model}

The present droplet model is simple, and is intended as a stepping stone towards a model describing a droplet size distribution. One particularly interesting option is the Quadrature Method of Moments (QMOM), as described by Marchiso et. al. (2003).

\section{Pipe roughness}

In the present model, the user has to specify a roughness height for the pipe wall. In reality, the presence of the film will influence the apparent wall roughness and thus the wall shear stress. This coupling may be included in later versions of the code.

\section{CONCLUSION}

A model for dilute gas-liquid flows with films at the walls has been implemented in the CFD code FLUENT using User-Defined Functions (UDF). The model consists of transport equations for droplets in the gas and for liquid transport in the film. Exchange of liquid between the two occurs by atomization of the film and by droplet deposition.

Predictions are in reasonable agreement with known cases, such as horizontal and vertical pipe flows. However, independent validation of the code is yet to be performed.

\section{REFERENCES}

CSANADY, G.T. (1963). "Turbulent diffusion of heavy particles in the atmosphere". J. Atmos. Sci., 20, 201.208.

LOTH, E. (2001). "An Eulerian turbulent diffusion model for particles and bubbles". Int. J. of Multiphase Flow, 27, 1051-1063.

MARCHISIO, D.L., VIGIL, R.D. and FOX, R.O. (2003). "Implementation of the quadrature method of moments in CFD codes for aggregation-breakage problems". Chemical Engineering Science, 58, 3337 3351

McCOY, D.D. and HANRATTY, T.J. (1997). "Rate of deposition of droplets in annular two-phase flow". Int. J. of Multiphase Flow, 3, 319-331.

MOLS, B., MITTENDORFF, I. and OLIEMANS, R.V.A. (2000)."Results from a two-dimensional turbulent diffusion-model for dispersion and deposition of droplets in horizontal annular dispersed gas/liquid flow", Int. J. of Multiphase Flow, 26, 6, 949-975.

MOLS, B., and OLIEMANS, R.V.A. (1998). "A turbulent diffusion model for particle dispersion and deposition in horizontal tube flow", Int. J. of Multiphase Flow, 24, 1, 55-75. 
PAN, L. and HANRATTY, T.J., (2002). "Correlation of entrainment for annular flow in vertical pipes", Int. J. of Multiphase Flow, 28, 3, 363-384.

TURTON, R. and CLARK, N.N. (1987), Powder Technol., 53, 127. 